\title{
A Numerical Model of a Bulk Flow TCP and its Routing Performance Analysis for the Optical IP Networks
}

\author{
V. Parthasarathy \\ Department of CSE, Chettinad College \\ of Engineering and Technology, NH-67 \\ Karur-Trichy Highways, Puliyur C.F. \\ Karur, Tamilnadu, India.
}

\author{
P. Anandakumar \\ Department of Information \\ Technology, Madras Institute of \\ Technology, Anna University Chennai, \\ Chennai, \\ Tamilnadu, India.,
}

\author{
V. Rajamani \\ Principal, Indra Ganesan College of \\ Engg., Manikandam, Tiruchirappalli, \\ Tamilnadu, India.
}

\begin{abstract}
A novel bulk flow TCP and its routing performance of the optical IP networks is analyzed numerically. Four types of data transmission viz. fixed size flow discard, fixed size early flow discard, variable size flow discard and variable size early flow discard for the optical domain are investigated. Various probability density functions for the data transmissions are derived for the computation. State transition diagrams for the various flow transmission techniques are designed to study the steady state probability of the buffer usage. The variable size early flow discard technique provides good throughput of $44 \%$ for a load of 3.5 for a mostly congested traffic where as the conventional packet discard exhibits only $3 \%$ of good throughput under same load and traffic condition. Buffer usage is found to be a minimum value for the variable size early flow discard $(43 \%)$ than the packet discard (99\%).
\end{abstract}

\section{Keywords}

Optical packet switching (OPS), Bulk flow TCP, round trip Time (RTT), variable-sized packets, wavelength conversion, optical router.

\section{INTRODUCTION}

Efficient data transport is very much important for the network to be effective particularly in wide-area applications. This scenario is applicable to all, ranging from web server to remote file, data systems to computational monitoring and even for super computers. This has been found to be a difficult task and often involves customization, requires in-depth technical knowledge in various areas like configuration of source, intermediate and destination nodes, optimization of TCP, buffering schemes, channelizing the data etc., Even then the complexity of making TCP to achieve high throughputs and efficient load balancing in the network with deep study on traffic engineering particularly for wide-area networks, achieving better congestion control mechanism are of major focus for the researcher to investigate [14].

In order to design an effective high-performance TCP/IP it is essential to study the performance of dynamics of TCP [5]. The research activities deal with a wide range of information for a solution towards excellent throughput, better quality of services, minimal delay and packet loss in TCP / IP are of major focus in reducing internet traffic. Conventionally the data are fragmented as packets and in some cases it is further fragmented as ATM calls.
However the objective is to arrive successfully in the desired destination without major loss or time to reconstruct the data. The practical issues that are to be solved for the conventional TCP are packets with different delay, out of order of packets, loss of packets etc. Conventionally the TCP experiences the issues like packet retransmission which ultimately consumes unnecessary network resource or this is done later than required, difficulty in calculating the round trip time (RTT) which will influence on the on the retransmission timer value, the TCP's congestion window cannot grow beyond a particular level due to RTT and hence this result in reduction of window overcrowding, even without any packet loss [6]. Most of the network resources are consumed by the use of various issues viz. Packet reordering, poor efficiency of the TCP, burstiness in traffic and TCP self clocking. In order to improve the performance of the system, modification in conventional TCP is very much essential to fulfill the requirements as mentioned and this is identified as one side issue for the data transfer technology. On the other side the everincreasing user demands and frequent updation in technologies for new applications [7] requires optimum potential. More than a decade, it is found that there is a vast growth in the Internet, large number of users making the internet facility for gaining information, communication purposes and entertainment. Similarly the enterprise users are relying mainly on the Internet for day to day business activities [8]. The reasons mentioned above all results in an exponential growth for the internet traffic. In the past decade the network bandwidth was consumed more [9]. This trend is also due to the emergence of multimedia applications, like video on demand, Internet telephony, interactive gaming and video conferencing etc., [10]. Thus a vast potential for bandwidth are very much essential inorder to solve the issues in telecommunications infrastructure.

This work proposes a novel technique which will solve the conventional problems identified in TCP oriented transmission and also a solution for wider bandwidth availability. By this method the TCP packets are consolidated as a single capsule and are termed as Bulk flow TCP and this flow is considered for routing and switching. By this method there is no necessity for the packet to reorder in the destination end and if there are ' $n$ ' packets in the flow, then the delay for the first packet will be the same for the $\mathrm{n}^{\text {th }}$ packet within the flow. More over the packet switching is very flexible and scalable as that of conventional routing and switching mechanism. The performance of the network like good throughput is found to increase by this method. For routing and switching operations, it is different from conventional packet switching technology. Similarly different discarding techniques are proposed for this work. 
The rest of the paper has been organized as follows: Section 2 deals about the proposed Bulk Flow TCP in detail. The routing concepts for the proposed Bulk Flow TCP are presented in section 3 . The performance evaluation for discarding techniques and throughput calculations are presented in section 4 . The results and discussions are presented in section 5. Conclusion on the work with further scope on research is presented in section 6 .

\section{BULK FLOW TCP}

The packets in the conventional TCP are consolidated as a single capsule to have a set of TCP packets which is termed as Bulk Flow TCP. Hence the processing time for every packet, packet delay, ordering of packets at the destination, routing and switching etc., are minimized into a uniform fashion.

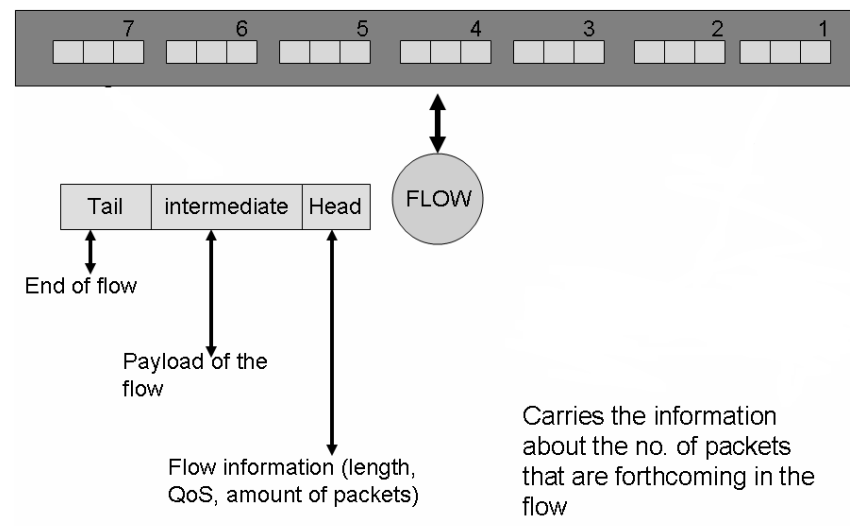

\section{Figure 1. Bulk Flow TCP}

Fig.1 describes the bulk flow TCP in which there are three components one is the head packet, another one is the intermediate packet and the third one is tail packet. The information about number of TCP packets within the flow, flow parameters, QoS parameters etc., are packed into the head packet. The intermediate packet will carry the content that is the data to be transmitted from source to the destination and the tail packet is to have the indication that the end of flow.

The parameters that are to be assumed for a flow like flow directionality as unidirectional, end point aggregation as doubleend point flows, protocol layer pertaining to specific port of network layer and flow timeout is fixed based on the delay tolerance of the first packet arrival [11].

\subsection{FLOW DISCARDING TECHNIQUES}

The bulk flow TCP when arrived at the buffer may not find space to accommodate and in such cases the discarding techniques like Flow Discard (FD) and Early flow Discard (EFD) are followed. The discarding operations as per Flow Discard (FD)are considered essential since the buffer has only limited space to accommodate and if the buffer is full, then any flow which comes after that will be discarded based on the flow discard method even if the packet contains control data or any other important information.

To avoid the problem as mentioned in the flow discard technique, the early flow discarding technique (EFD) introduces a parameter called thresh-hold value ' $K$ '. This threshold value is decided based on the buffer size and for every arrival of the flow is to be calculated for average queue size and then the flow is allowed only if it falls below the thresh hold value otherwise the incoming flow is studied for its importance and if it has control data or any other important data it is allowed or simply discarded.

Here the flow length is decided by referring to the bandwidth availability, other network resources, delay tolerance etc., This paper considers two possibility of flow size. In one case the size of the flow is assumed to be constant irrespective of the data to be transmitted and hence the flow is padded with dummy data inorder to have a fixed size for the flow. In other case the flow is to have variable size and hence based on the number and size of incoming packet and it is converted as flow.

Based on the conventional packet discarding techniques and the proposed flow classifications the following four discarding techniques are proposed:

1. $\quad$ Fixed sized Flow Discard (FFD)
2. $\quad$ Fixed sized Early Flow Discard (FEFD)
3. $\quad$ Variable sized Flow Discard (VFD)
4. $\quad$ Variable sized Early Flow Discard (VEFD)

\section{MULTI DOMAIN SOLUTION FOR OPTICAL FLOW ROUTING}

In order to give solution for a successful transmission of flow from source to the destination, the routing techniques are to be investigated to a longer length.

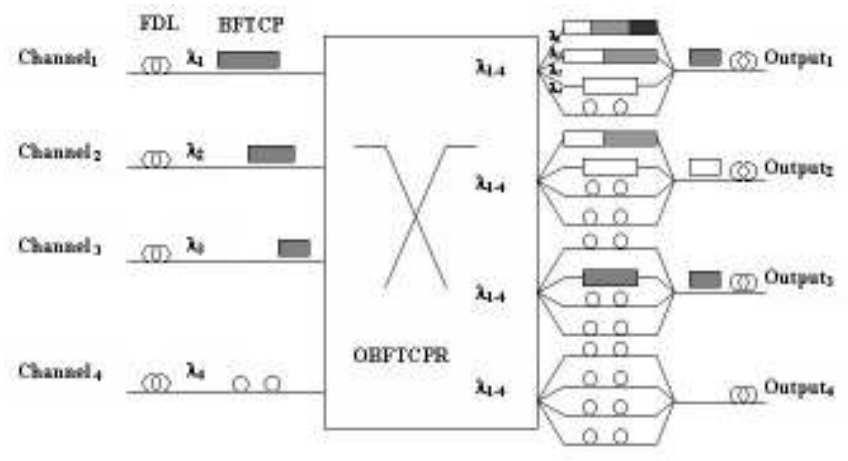

Figure 2. Optical flow Routing

The conventional solutions for Packet routing can also be applied to flow routing as follows:

1. Time domain optical flow routing (TDOFR)

2. Wavelength domain optical flow routing (WDOFR) and

3. Space domain optical flow routing (SDOFR)

\subsection{Time Domain Optical Flow Routing}

In time domain optical flow routing the optical buffering takes a dominant role while the flow could not be transmitted over a desired channel. By this method each channel is to adopt with fiber delay lines (FDL) in order to have buffering either of ingress level or at egress level and in some cases both. The flow or the packet for the case of input to the router are allowed to enqueue in the buffer based either on flow discarding or early flow discarding technique and thus it will gain time before getting its turn to transmit to the destination. 
The main drawback here is the maintenance of recording status of each flow which enters into the FDL. This ultimately requires a complex set of procedure. In addition to this to upkeep the order of the flow and uniform delay the complexity of the network control is further to be increased and provision on random access to buffer is ensured. The fiber delay line indicating the buffer functionalities are shown in Fig.2

\subsection{Wavelength Domain Ofr}

The optimal solution, which helps in most of the times while the flow experiences the congestion, is to go for wavelength conversion. The wavelength conversion will provide buffer for the flow to reach the destination at the same output port. To illustrate this let us assume that few flows are to be transmitted to the destination, the first flow with the first wavelength will go through the desired path, the second flow cannot take the same wavelength if the corresponding wavelength is full. In such cases the second flow is converted to another wavelength and then the flow will continue to travel in the same channel towards destination as shown in Fig.2. Moreover the contention resolution is also handled well in wavelength domain. However the main drawback here is that the order of the flow at the destination will not be the same as that of the source and hence the reordering of flow at the destination is required.

\subsection{Space Domain Ofr}

In order to have redundancy of reaching destination, provision of multiple paths or channels to reach the destination is insisted. With reference to the wavelength converted routing; the flow can continue to travel in the same channel with a converted wavelength. If the existing channel does not have any free wavelength, then change of channel (path) is required. It has four different channels and each channel has got four wavelengths. Hence if the channell exhaust with all available wavelengths then ultimately the flow is transmitted to the second channel. In most of the cases the channel wavelength need not be changed. Even though the flow order is not maintained and the corrupted flow is received, the successful transmission is ensured here.

\section{ANALYSIS ON TRAFFIC MODEL, BUFFER USAGE, STEADY STATE PROBABILITY THROUGHPUT}

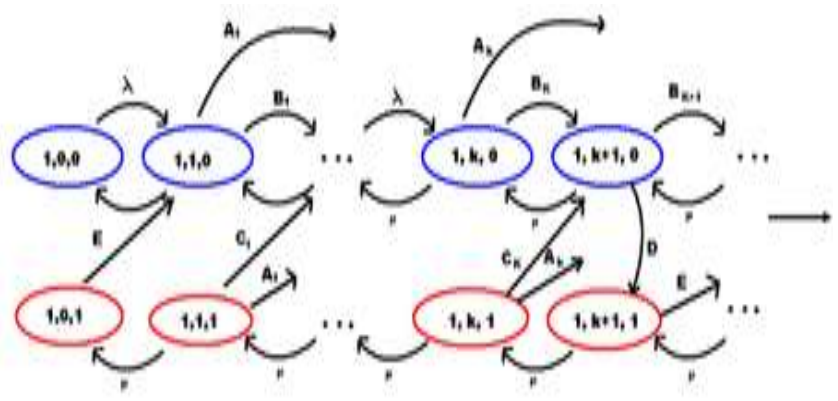

Figure 3. Wavelength converted variable size flow discard's state transition diagram

The state transition diagram for variable size flow discard is represented in Fig.3. Here the non discarding mode is represented in the top row and the discarding mode is represented in the bottom row. The forward direction arrow represents the arrival of bulk flow TCP and the reverse arrow represents the servicing the flow in buffer queue. The various parameters to represent the packet arrival rates and the nature of the packet in the flow are as follows:

$A_{i}$ - Head of new Variable size flow such that Number_of_Packets $>(B-i) . \mu$

$\mathrm{B}_{\mathrm{i}}$ - Head of new Variable size flow such that Number_of_Packets $<(B-i) \cdot \mu$

$\mathrm{C}_{\mathrm{i}}$ - Head of new Variable size flow such that Number_of_Packets $<(B-i) \cdot \mu($ or $) L$

$\mathrm{D}_{\mathrm{i}}$ - Packet is not a head of new Variable size flow

$E_{i}$ - Head of new Variable size flow such that Number_of _Packets $<L$

\subsection{Traffic Model}

The study on the network traffic is being carried out based on the probability of a particular packet to successfully transmit in the network. The probability density function of the proposed bulk flow TCP which consists of i packets for the variable size can be expressed by [13]:

$$
P\{x=i\}= \begin{cases}\frac{q(1-q)^{i-1}}{\sum_{j=1}^{L} q(1-q)^{j-1}} & \text { if } i \leq L \\ 0 & \text { if } i>L\end{cases}
$$

Here, $\mathrm{q}$ is the distribution of the number of packets per flow which is assumed as a geometric function [12] and L is the maximum number of packets in a flow. In this analysis, we have taken $\mathrm{L}$ is 7 . The probability density function for the fixed size flow by assuming seven packets in a flow can be written as

$$
P\{x=i=7\}=\frac{q(1-q)^{6}}{\sum_{j=1}^{7} q(1-q)^{j-1}} \text { for } \mathrm{i}=\mathrm{L}
$$

The number of packets in a flow is related to the buffer depth B and packet parameter $\mu$ such that $L / \mu \leq B$. The contention occurs in the traffic, if the ratio of packet arrival rate to the packet parameter is greater than $\mathbf{1}$. The total packet arrival rate is given by the packet arrival rate $\lambda$ and the total number of wavelength $\mathrm{n}_{\mathrm{w}}$ The load per wavelength has been measured using the expression

$\rho=\frac{\lambda}{\mu}$.

The packet arrival rate can be calculated for both fixed size flow and variable size flow as follows: 
Packet arrival rate for variable sized flow

$$
\lambda=\lambda^{1}\left[1-q(1-q)^{L}\right]
$$

Packet arrival rate for fixed sized flow

$$
\lambda=\lambda^{1}\left[1-q(1-q)^{7}\right]
$$

Where, $\lambda^{1}$ is the Poisson's packet arrival rate.

\subsection{Buffer Usage}

The utilization of buffers for the flow or the packet in the optical network is an important analysis. Buffers are normally represents the fiber delay line and the technology towards optical buffering have not yet grown to the stage of storing higher capacity for a longer duration. For a successful implementation of optical network is that the minimum utilization of buffers. The usage of buffer for the proposed bulk flow TCP can be calculated using equation (6) and (7) for the variable size flow and fixed size flow respectively.

Variable size flow $U=\frac{\sum_{i=0}^{B} i . P(Q=i)}{B}$

Fixed size flow $U=\frac{\sum_{I=0}^{B} I . P(Q=I)}{B}$

where, $I=7 l, l$ is an integer to represent the number of flow in the buffer and this will increase such that $(7 l) \leq \mathrm{B}$

\subsection{Steady State Probability Analysis}

When the data is transmitted over the fiber along with the delay lines the probability for the packet to occupy a particular wavelength $(\mathrm{m})$ out of available wavelengths $\mathrm{n}_{\mathrm{w}}$, space in the buffer (i) out of the buffer capacity ' $B$ ' and probability for successful transmission without drop (f) can be derived under steady state for the conventional packet discard and there from the probability for fixed size flow discard, variable size flow discard, fixed size early flow discard and variable size early flow discard are be derived as follows:

\section{Packet discard}

The steady state probability of buffer occupancy for optical packet routing with packet discard is written as

$$
P_{n_{w}, B, f=1}+\sum_{m=1}^{n_{w}} \sum_{i=0}^{B} P_{m, i, f=0}=1
$$

where, $m$ is the wavelength variable, $n_{w}$ is the total number of wavelength available, $i$ is the buffer space variable, $B$ is the buffer depth or buffer capacity, $\mathrm{f}=0$ non represents discarding mode and $\mathrm{f}=1$ represents discarding mode

\section{Flow Probability}

The steady state probability $\left(\mathrm{P}_{\mathrm{m}, \mathrm{i}, \mathrm{f}}\right)$ of a variable size flow can be computed as

$$
\sum_{m=1}^{n_{w}} \sum_{i=0}^{B} \sum_{f=0}^{1} P_{m, i, f}=1
$$

And for the fixed size flow, the steady state probability $\left(P_{m, I, f}\right)$ is

$$
\sum_{m=1}^{n_{w}} \sum_{I=0}^{B} \sum_{f=0}^{1} P_{m, I, f}=1
$$

The good throughput $(\mathrm{G})$ can be computed as

$$
\begin{gathered}
\text { Good_Throughput }(G)=\frac{\text { Successful_transmission_of_packets_in_a_flow }}{\text { Total_flow_arriveat_the_i/p_router }} \\
=\frac{\text { non_corrupted_flow }}{\text { Total_flow }} \\
=\frac{\sum_{m=1}^{n_{w}} \sum_{I=0}^{B} \sum_{f=0}^{1} n \cdot P(m, i, f, v=1)}{\sum_{m=1}^{n_{w}} \sum_{I=0}^{B} \sum_{f=0}^{1} n \cdot P(m, i, f)} \\
\sum_{m=1}^{n_{w}} \sum_{I=0}^{B} \sum_{f=0}^{1} n \cdot P(m, i, f, v=1) \\
\sum_{n=1}^{L} n \cdot \frac{q(1-q)^{n-1}}{\sum_{j=1}^{L} q(1-q)^{j-1}}
\end{gathered}
$$

where, $\mathrm{v}$ is 1 , the transmission of flow gives good throughput otherwise corrupted flow, $\mathrm{n}$ is the flow length in time slots and $\mathrm{w}$ is the length of an arriving variable flow size.

The throughput for the various packets or flow can be calculated based on the conditional probability $(\mathrm{C})$ values. The conditional probabilities for the Packet Discard (PD), Fixed size flow discard (FSFD), Variable sized flow discard (VSFD), Fixed size early flow discard (FSEFD) and variable size early flow discard (VSEFD) are as follows.

\section{$\underline{\text { Packet Discard (PD) }}$}

The conditional probability $\left(C_{n, m, i}^{P D}\right)$ for the packet discard will be

$$
C_{n, m, i}^{P D}=\left\{\begin{array}{c}
1 \text { if } 0 \leq i \leq B-(n / \mu) \text { and } 1 \leq m \leq n_{w} \\
\text { or } i=B \text { and } 1 \leq m \leq n_{w} \\
0
\end{array}\right.
$$

Supposing that a departure occurs before an arrival then the probability will be 


\section{Variable Size Flow Discard (VSFD)}

The conditional probability for the Variable size flow discard $\left(C_{n, m, i}^{V S F D}\right)$ can be written as

$C_{n, m, i}^{V S F D}=\left\{\begin{array}{c}1 \text { if } 0 \leq i \leq B-(n / \mu) \text { and } 1 \leq m \leq n_{w} \text { or } i=B \text { and } 1 \leq m \leq n_{w} \\ \text { or } B-(n / \mu)+1 \leq i \leq B \text { and } m<n_{w} \\ 0 \text { if } B-(n / \mu)+1 \leq i \leq B \text { and } m=n_{w} \text { or } i=B \text { and } m=n_{w}\end{array}\right.$

\section{Fixed Size Flow Discard (FSFD)}

The conditional probability for the Fixed size flow discard $\left(C_{n, m, i}^{F S F D}\right)$ is

$$
C_{n, m, i}^{F S F D}=\left\{\begin{array}{c}
1 \text { if } 0 \leq I \leq B-(n / \mu) \text { and } 1 \leq m \leq n_{w} \text { or } I=B \text { and } 1 \leq m \leq n_{w} \\
\quad \text { or } B-(n / \mu)+1 \leq I \leq B \text { and } m<n_{w} \\
0 \text { if } B-(n / \mu)+1 \leq I \leq B \text { and } m=n_{w} \text { or } I=B \text { and } m=n_{w}
\end{array}\right.
$$

\section{Variable Sized Early Flow Discard (VSEFD)}

To calculate the conditional probability for the Variable Size Early Flow Discard $\left(C_{n, m, i}^{V S E F D}\right)$ for the fixed threshold value can be written as

$$
C_{n, m, i}^{V S E F D}=\left\{\begin{array}{c}
C_{n, m, i}^{P D} \text { if } i<k \text { and } 1 \leq m \leq n_{w} \\
C_{n, m+1,0}^{V S E D} \text { if } i \geq k \text { and } 1 \leq m<n_{w} \\
0 \text { when } i \leq k \text { and } m=n_{w}
\end{array}\right.
$$

\section{Fixed Sized Early Flow Discard (FSEFD)}

The conditional probability for the Fixed Size Early Flow Discard $\left(C_{n, m, i}^{F S E F D}\right)$ is

$$
C_{n, m, i}^{F S E F}=\left\{\begin{array}{c}
C_{n, m, i}^{P D} \text { if } i<k \text { and } 1 \leq m \leq n_{w} \\
C_{n, m+1,0}^{V S F D} \text { if } i \geq k \text { and } 1 \leq m<n_{w} \\
0 \text { when } \mathrm{I} \leq k \text { and } m=n_{w}
\end{array}\right.
$$

\section{RESULTS AND DISCUSSIONS}

The analysis of network traffic for the proposed bulk flow TCP and the routing are studied for the discarding techniques. This analytical model for bulk flow TCP is a tool to study the performance of the network traffic in the optical environment. The various schemes like PD, FSFD, VSFD, FSEFD, and VSEFD are compared to each other and optimum solutions have been

obtained. The network traffic is assessed by viewing the impact on certain traffic parameters like packet arrival rate $\lambda$, the flow parameter $q$ and the maximum number of packets per flow $L$. For the simulation purpose, the parameters used for computation have been presented below.

The packet parameter, $\mu$ is 1 , depth of buffer $\mathrm{B}$ (in time slots) is 70 , the threshold value of Buffer $\mathrm{k}$ (in time slots) is 28 .

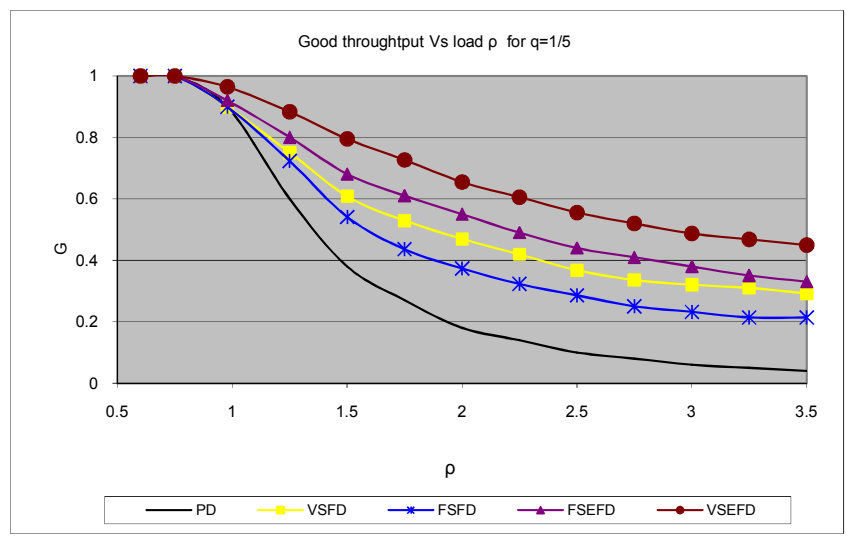

Figure 4. Good-throughput $G$ vs. load $\rho$ for FSEFD, FSFD, VSEFD, VSFD and PD, $\mu=1, B=70, k=28, L=7$ and $\mathrm{q}=1 / 5$

The study on the packet arrival rate $\lambda$ with the packet parameter $\mu=1$ on the good throughput $G$ is depicted in Fig 4. Here, the load $\rho$ varies from 0.5 to 3.5 and packet arrival rate $\lambda$ also varies in the same range. It is seen from the figure that the good throughput $G$ decreases with the increase of $\lambda$ irrespective of the PD, FSFD, VSFD, FSEFD, and VSEFD. However, good throughput $G$ for PD drops in a faster rate than with the other method. The response is well recognized for the higher loads that is when $\lambda$ is more than 1 or this is called as period where the congestion being build-up. From the figure it can also seen that the load is 3.5, the good throughput for the PD is only 3\%. This is due to the congestion at this point is very high. Even though the congestion is very high at this load, the flow based transmission provides higher good throughput values.

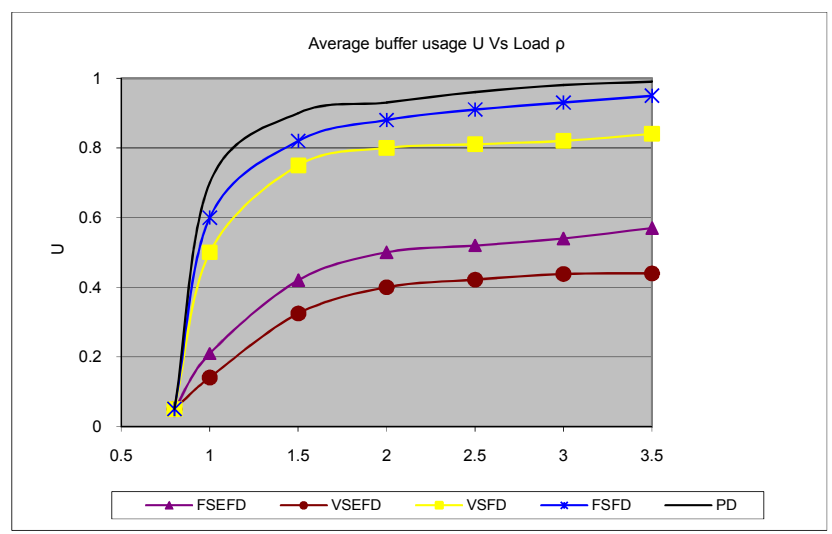

Figure 5. Average buffer usage $U$ vs. load $\rho$ for FSEFD, FSFD, VSEFD, VSFD and PD, $\mu=1, B=70, L=7, \mathrm{k}=28$ and $\mathbf{q}=\mathbf{1} / \mathbf{5}$. 
They are $21 \%$ for FSFD, $30 \%$ for VSFD, 32\% for FSEFD and $44 \%$ for VSEFD. It is thus proved that the variable size early flow discard outperforms over other flow discarding methods. The result also reveals that the conventional packet discarding technique ends up with large percentage of corrupted flow during congestion and the variable size early flow discard shows excellent result even during congestion period (44\%).

Fig.5 shows the usage of buffer for various flow discarding policies. The buffer usage can be calculated by

$$
U=\frac{\sum_{i=0}^{B} i P_{m, i, f}}{B}
$$

The buffer usage value $U$ for both EFD methods is limited to a minimum value of $60 \%$. This is due to the following reasons. From the figure it is seen that the packet discard technique utilized higher values of buffer. This is due to the fact that there is no flow orientation in the traffic. In the case of flow discarding methods viz. FSFD and VSFD, the buffer usage values are higher when the average flow length is longer.

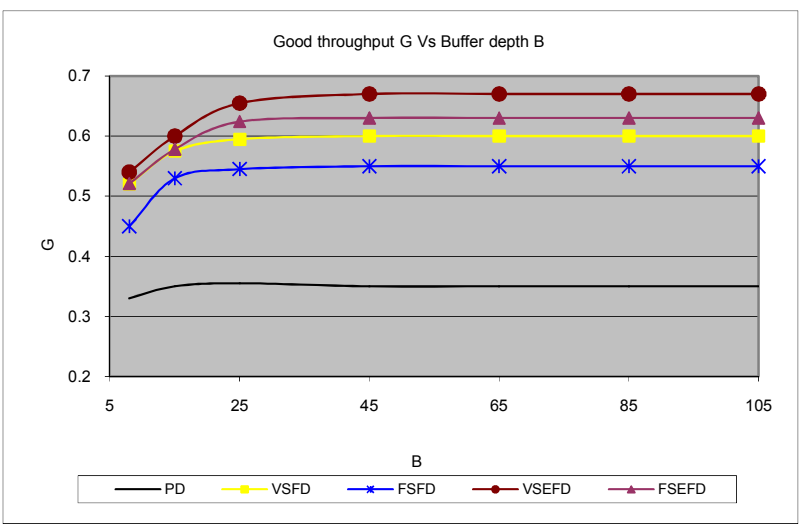

Figure 6. Good-throughout $G$ vs. buffer depth $B$. $\mu=1$,

$$
L=7, q=1 / 5, k=[B / 2] \rho=1.5
$$

However, for the early flow discarding methods viz. FSEFD and VSEFD, these techniques occupy less buffer utilization even when the average flow length are longer. From this it is clear that the utilization of buffer helps us to choose which techniques has been adopted to implement the network traffic.Good throughput with buffer depth is presented in Fig. 6. Here, we have simulated the performance of good throughput for the system level parameter such as service rate $\mu$ with respect to various flow techniques.

Here, VSEFD and FSEFD attain a saturation level $67 \%$ and $63 \%$ respectively of good throughput. But in the case of VSFD and FSFD attain the saturation level has attained $60 \%$ and $55 \%$ respectively of good throughput. The saturation level for PD is only $35 \%$. This is due to the corrupted flow for the packet based transmission. It is also seen that VSEFD technique provides highest good throughput and the PD techniques provides lesser value of throughput. These results are helpful for designing the optical network router. The performance of threshold $\mathrm{k}$ with respect to good-throughput $G$ is depicted in Fig.7 for different $\lambda$ of $1.1,1.4$ and1.7. From the figure it is seen that the good throughput $G$ does not respond in a faster rate for the change in the value of threshold $k$.

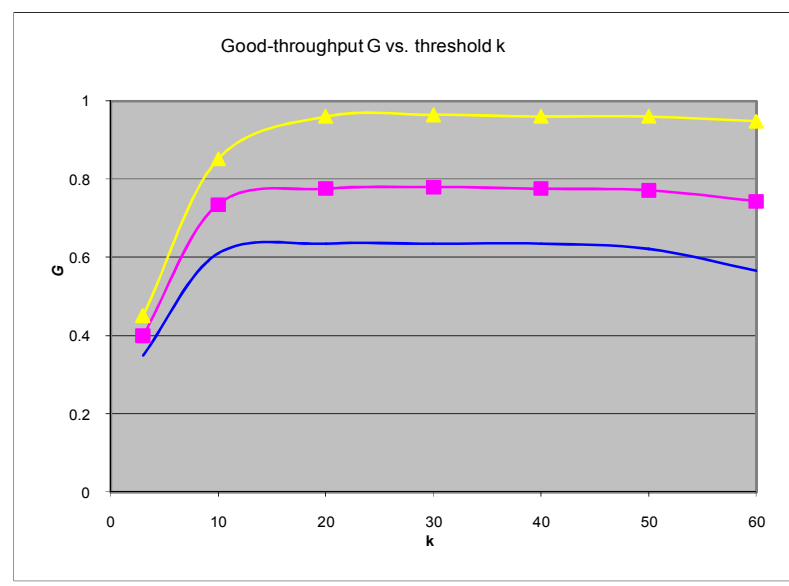

\section{Figure 7. Good-throughput $\boldsymbol{G}$ vs. threshold $\boldsymbol{k}$ for VSEFD under different $l, q=1 / 5, \mu=1, B=70, L=7$.}

It is also seen that the threshold values inbetween 10 to 50 , good throughput values are almost constant. But if the threshold values are below 10, the good throughput values are changing drastically.

\section{CONCLUSIONS}

A model of bulk flow TCP for the optical IP network has been implemented numerically. Various data transmission techniques viz. FSFD, FSEFD, FSEFD and VSEFD have been studied to find the probability density function. Steady state diagram have been derived for the various transmission modes. Variable size early flow discard provided a maximum good throughput of $44 \%$ for a peak load condition. Buffer utilization was very less against conventional packet discarding method. It has been observed from the numerical study that for the increase in buffer depth, good throughput increases to maximum of $67 \%$ for a variable size early flow discard.

Variable size early flow discard exhibited a higher throughput than other data transmission methods for a variation in the threshold value. This numerical study on Bulk flow TCP with routing concepts suggested that the variable size early flow discard technique as a best method to implement in the optical domain. This technique can also be tried for simulation with a help of simulator tool and the results can be compared for the use in real time applications and also this will provide a tool for the network design engineer.

\section{REFERENCES}

[1] Ning Zhang and Hong Bao," Load and traffic engineering in optical network" Computer science and Information Technolgoy, 2009, ICCSIT 2009. $2^{\text {nd }}$ IEEE International Conference on 8-11 Aug. 2009 pp.360-363.

[2] M. Hassan and R. Jain "High Performance TCP/IP Networking: Concepts, Issues, and Solutions". Prentice Hall, 2004. 
[3] D. Kitabi, M. Handley, and C. Rohrs "Congestion control for high bandwidth-delay product networks". In Proc. of SIGCOMM. 2002.

[4] N. S. V. Rao, J. Gao, and L. O. Chua "On dynamics of transport protocols in wide-area internet connections", In L. Kocarev and G. Vattay, editors, Complex Dynamics in Communication Networks. Springer- Verlag Publishers, 2005.

[5] D.P.Hong, C.Albuquerque, C.oliveria and Tsuda, "Evaluating the impact of emerging streaming media applications on TCP/IP performance," IEEE comm.. Mag., vol, 39, pp 76-83, 2001.

[6] S. Floyd, "A report on recent developments in TCP congestion control,” IEEE Commun. Mag., vol. 39, pp. 84-90, 2001.

[7] G. Ge, L. Alan, and C. SIAM. "On the existence of designs with application to optical network," Journal on Discrete Mathematics, 2007, 21, pp. 851-864.

[8] D. O. Awduche and B. Jabbari. "Internet Traffic Engineering using Multi- Protocol Label Switching (MPLS)," Computer Networks, 2002, (40): pp. 111-129.
[9] K. Kar, M. Kodialam, and T.V. Lakshman. "Minimum Interference Routing of Bandwidth Guaranteed Tunnels with MPLS Traffic Engineering Applications," IEEE Journal on Selected Areas in Communications, 2000, 18(12):pp. 2566-2579.

[10] B. Wang, X. Su, and C.P. Chen. "A New Bandwidth Guaranteed Routing Algorithm for MPLS Traffic Engineering," In Proceedings of ICC, New York - USA, 2002, Vol. 2, pp. 10011005.

[11] C. Lam and D. Simeonidou, "The application of optical packet switching in future communication networks," IEEE J. Sel. Areas Commun., Aug. 2004.

[12] Y. Lapid, R. Rom and M. Sidi, Analysis of discarding policies in high-speed networks, IEEE Journal on Selected Areas in Communications, vol. 16, no. 5, (1998), pp. 764-777.

[13] Jenny J. He and Dimitra Simeonidou, "On WavelengthConverted Optical Routers

Employing Flow Routing", Journal of Lightwave Technology, Vol. 23, No. 3, March 2005, pp-972-981 